\title{
Down-Regulation of MicroRNA-214 Contributed to the Enhanced Mitochondrial Transcription Factor A and Inhibited Proliferation of Colorectal Cancer Cells
}

\author{
Kaiming Wu Jun Ma ${ }^{\mathrm{a}}$ Yanfeng Zhan ${ }^{c}$ Kuanzhi Liu ${ }^{\mathrm{d}}$ Ziyin Ye Jianhui Chen ${ }^{\mathrm{a}}$ \\ Kaiwu $\mathrm{Xu}^{\mathrm{a}}$ Hongli Huang ${ }^{f}$ Yulong $\mathrm{He}^{\mathrm{a}}$
}

\begin{abstract}
aDepartment of Gastrointestinal Surgery Center, First Affiliated Hospital of Sun Yat-Sen University, Guangzhou, bepartment of Thoracic Surgery, First Affiliated Hospital of Sun Yat-Sen University, Guangzhou, 'Department of Obstetrics and Gynecology, First Affiliated Hospital of Sun Yat-Sen University, Guangzhou, dDepartment of Anaesthesiology, First Affiliated Hospital of Sun Yat-Sen University, Guangzhou, eDepartment of Pathology, First Affiliated Hospital of Sun Yat-Sen University, Guangzhou, fDepartment of Gastroenterology, Guangzhou Digestive Disease Center, Guangzhou First People's Hospital, Guangzhou Medical University, Guangzhou, China
\end{abstract}

\section{Key Words}

Colorectal cancer $・$ TFAM $\bullet$ miR-214 $•$ Proliferation $・ N F-k B$

\begin{abstract}
Background/Aims: Colon cancer, also known as colorectal cancer (CRC), is one of the most common malignant tumors globally. Although significant advances have been made for developing novel therapeutics, the mechanisms of progression of colorectal cancer are still poorly understood. Methods: In this study, we identified down-regulation of microRNA-214 (miR-214) as the contributing factor for CRC. Mitochondrial transcription factor A (TFAM) and miR-214 expression in tumor samples from colorectal cancer patients and cancer cell lines were examined by reverse transcription and real-Time PCR (qPCR) or Western Blotting. Results: Our data demonstrated that miR-214 was significantly down-regulated in the tissue samples from CRC patients as well as CRC derived cell lines. TFAM overexpression was also observed in CRC patients and identified as a target for miR-214. Knockdown of TFAM by miR214 mimics significantly inhibited the proliferation of CRC cell lines. Also, down-regulation of TFAM inhibited nuclear factor kappa-light-chain-enhancer of activated B cells (NF-KB) nuclear translocation and the expression of NF- $\mathrm{kB}$ depended genes. Conclusion: In conclusion, our data suggested that down-regulation of MiR-214 contributed to the enhanced TFAM expression and decreased proliferation of CRC cells.

(C) 2018 The Author(s)

$\mathrm{K}$. Wu, J. Ma and Y. Zhan contributed equally to this work.




\section{Introduction}

Colon cancer, also known as CRC, which originated from epithelial cells of colon or rectum, is one of the most common malignant tumors world wide [1-3]. The abnormal growth of cancer cells results in invading or spreading of cancer cells to other parts of the body. The exact causes of colon cancer are still illusive since more than $75 \%$ of colon cancer patients carry little or no genetic risk [4]. Although significant advances have been made for developing novel therapeutics, colon cancer remains an incurable disease mainly due to the multidrug resistance (MDR) for chemotherapy agents [5]. With surgical resection and aggressive chemotherapy, 50\% of colorectal carcinoma patients still develop recurrent disease [1]. Tumor progression and tumor cell metastasis are a multi-step process involving in different mechanisms such as accelerated proliferation of cancer cells, the interaction between cancer cells and the extracellular matrix (ECM). Therefore, further understanding of the molecular mechanism in colon cancer is needed for developing new therapeutic approaches to improve the current chemotherapeutic regimen.

MicroRNAs (miRNAs) are a class of non-coding short RNA molecules which negatively regulate gene expression at the post-transcription level via binding with 3' untranslated region (3'UTR) of mRNAs [6]. Since the targets of a single miRNA may be multiple, abnormal expression of miRNAs during the development of cancer as well as the disease progression have been reported [7]. miRNAs have been identified as a novel post-transcription mechanism for regulating gene expression [6] and reported to play important roles in regulation of cell proliferation, migration, and differentiation [6]. Current studies identified that several miRNAs such as let-7 [8], miR-143 and miR-126 [9, 10] which were involved in colon cancer. Furthermore, miRNAs circulating in blood were proposed as the potential biological markers for colon cancer diagnosis [11], implying indispensable roles of miRNA in the prognosis of colon cancer.

TFAM, a high-mobility group (HMG) protein, plays a central role in mitochondrial DNA (mtDNA) replication, transcription and inheritance. TFAM is reported to be associated with tumorigenesis in breast, cervical, prostate, small cell lung cancers [12-16]. Also, frequent frameshift mutations in the coding mononucleotide repeat of TFAM was demonstrated in sporadic CRC derived cell lines and CRC tissues $[17,18]$. Previous study suggested that the reduced mtDNA copy number increased the sensitivity of tumor cells to chemotherapeutic drugs, suggesting that TFAM could be a potential contributor for tumor progression [19]. However, little is known in regarding the posttranscriptional regulation of endogenous TFAM in CRC.

The relationship between miR-214 and TFAM was previously reported. miR-214 was considered to act as a tumor suppressor and its overexpression significantly suppressed the proliferation and migration of cervical cancer Hela and Caski cells by targeting TFAM $[15,20]$. In this study, we identified the miR-214 as a novel regulator for CRC. Furthermore, downregulation of miR-214 was observed in the tumor samples obtained from CRC patients and CRC cells. Further analysis confirmed that miR-214 inhibited proliferation in CRC cells via the up-regulation of TFAM.

\section{Materials and Methods}

Ethics statement, patients and samples

Tissue samples of CRC patients who underwent resection of their tumors at the Gastrointestinal Surgery Center, the first affiliated hospital of Sun Yat-Sen University, were prospectively obtained for this study by following institutional review board guidelines. This study was reviewed and approved by the 


\section{Cellular Physiology Cell Physiol Biochem 2018;49:545-554 and Biochemistry Published online:29August, $2018 \begin{aligned} & \text { DOI: 10.1159/000492992 } 2018 \text { The Author(s). Published by S. Karger AG, Basel } \\ & \text { www.karger.com/cpb }\end{aligned}$ \\ Wu et al:: MicroRNA-214 Targeting TFAM}

Research Ethics Board of Sun Yat-Sen University. All participating subjects were formally informed for the purpose of using the samples and a letter of consent was signed by every subject involved.

\section{Cells, miR-152 mimics and chemicals}

Normal colon cell line CCD-18Co (ATCC $®$ CRL-1459 ${ }^{\mathrm{Tm}}$ ), human colon cancer cell line HCT- 116 (ATCC®CCL-247 ${ }^{\mathrm{TM}}$ ), HT-29 (ATCC®HTB-38 ${ }^{\mathrm{TM}}$ ), SW620 (ATCC $® C C L-227^{\mathrm{TM}}$ ), Caco-2 (ATCC $® H T B-37^{\mathrm{TM}}$ ) and RKO ATCC $\AA C R L-2577^{\text {TM }}$ were purchased from American Type Culture Collection (ATCC). Human colon cancer cell line DIFI was purchased from Bioleaf Corporation (Shanghai, China). Colon cancer cell line CW-2 cells were stored in our laboratory. All cell lines were maintained in Dulbecco's Modified Eagle Medium (DMEM, Gibco, Carlsbad, CA, USA) supplemented with $10 \%$ fetal bovine serum (FBS) (Gibco, Carlsbad, CA, USA).

The putative miRNA binding sites for TFAM 3'UTR were screened by using TargetScan (http://www.targetscan.org/, Release 7.0, August 2015). The MISSION $®$ microRNA Mimic hsa-miR-214, scramble control of miRNA mimic and miR-214 inhibitor were commercially purchased from SigmaAldrich (St. Louis, MO, USA). Transfection of cell lines with plasmid DNA or microRNA mimic was conducted by using Lipofectamine2000 (Invitrogen, Carlsbad, CA, USA ) according to the instructions of the manufacturer.

Reporter plasmid and luciferase based miRNA function assay

Human TFAM 3'UTR and mutated TFAM 3'UTR were artificially synthesized by Genescript (Nanjing, China) and cloned to the reporter plasmid psiCHECK2 (Promega, Madison, WI, USA). Luciferase activities in the different cell lines transfected with psiCHECK2-PTEN-3'UTR along with miR-214 mimic or scramble control were measured by Dual-Glo® Luciferase Assay System (Promega Corp., Madison, WI, USA) following the instruction of manufacturer. The luminescence signal of luciferase was measured by a VICTORX5 ${ }^{\mathrm{TM}}$ Multilabel Counter (Perkin-Elmer, Waltham, MA, USA). Relative percentages of luciferase activity were calculated by comparing with a scramble control transfected cells.

\section{qPCR}

TRziol Reagent (Invitrogen, Carlsbad, CA, USA) was used for total RNAs isolation from in vitro cultured CRC cells as well as tissue samples obtained from CRC patients. The cDNA generation for quantification of miRNAs expression was conducted by using Hairpin-it ${ }^{T M}$ miRNA RT-PCR Quantitation kit (GenePharma, Shanghai, China) and MystiCq ${ }^{\circledR}$ microRNA qPCR Assay Primer (Sigma-Aldrich, St. Louis, MO, USA) according to manufacturer's instruction. For the evaluation of the cellular gene level, the cDNAs of in vitro cultured cells or tissue samples were obtained via reverse transcription by AMV reverse transcriptase (Promega Corp., Madison, WI, USA). A combination of oligo dT and random hexamer was used for cDNA synthesis. The qPCR detection with SYBR Green Mix (Life technologies Crop., Carlsbad, CA) for measuring the indicated genes and miR-214 expression was described previously $[21,22]$. The transcript level of human RPL32 and U6 were also monitored from the same sample to serve as reference genes for normalization. The relative gene expression was quantified by the $2^{-\Delta \Delta C T}$ method as previously described [23]. Primers used in this study were listed as Table 1.

Flow cytometry based cell cycle assay

A total of $1 \times 10^{6} \mathrm{CRC}$ cells in each group were fixed with $70 \%$ ethanol and permeabilized by phosphate buffered saline (PBS) containing 0.5\% TritonX100 along with the treatment with DNase-free RNase A (Sigma-Aldrich, St. Louis, MO, USA). Then the fixed cells were stained by propidium iodide for cell cycle analysis by flow cytometry machine (FACS Calibur, BD Bioscience, San Jose, CA, USA).

\section{Western Blotting}

Western Blotting was conducted as previously described [24]. Briefly, after denatured proteins were transferred into polyvinylidene difluoride (PVDF) membrane, the membrane was blocked and probed by rabbit anti-TFAM antibody (dilution 1:3000, Santa Cruz Biotechnology, Santa Cruz, CA, USA), or rabbit antiNF- $\kappa$ B p65 (dilution 1:3000, Santa Cruz Biotechnology, Santa Cruz, CA, USA) for overnight at $4^{\circ} \mathrm{C}$. Specific reactions between different antibodies and their corresponding targets were detected by using goat antirabbit conjugated with horseradish peroxidase (dilution 1:5000, Sigma, St. Louis, MO, USA) and revealed by a chemiluminescence substrate (Thermo Fisher Scientific, Waltham, Massachusetts, USA). The membrane was also probed with anti-Tubulin antibody (dilution 1:3000, Santa Cruz Biotechnology, Santa Cruz, CA, 
USA) to normalize the total protein loading. The chemiluminescence signal was digitally recorded and analyzed by the ChemiDoc X-ray spectrometer (XRS) imaging system (Bio-Rad, Hercules, CA, USA) with ImageLab (Version 6.1, Bio-Rad).

\section{Clonogenic cell survival assay}

Clonogenic cell survival assay was conducted as previously described with modifications as well [25]. Briefly, miR-214 mimic, miR-214 inhibitor or scramble control transfected cells were trypsinized and counted for viable cells. Totally $1 \times 10^{4}$ cells were seeded into $100 \mathrm{~mm}$ cell culture dishes and maintained for one week. Then the cell colonies were stained with gentian violet for pictures.

\section{Statistical analysis}

The statistical analysis was conducted and analyzed using One-way analysis of variance (ANOVA) in statistics package for social science (SPSS, Version 16.0, Inc., Chicago, IL, USA). Differences in indicators between treatment samples, such as luciferase activity, miR-214, TFAM mRNA and other gene levels were assessed by Student $t$-test. A two tailed P-value of less than 0.05 was considered significant.

\section{Results}

The miR-214 expression level was reduced in CRC patients and CRC cell lines

As demonstrated in Fig. 1A, compared with the adjuvant healthy tissues, the expression of miR-214 was significantly reduced in paired CRC tissues, suggesting a down-regulation of miR-214 in CRC $(\mathrm{P}<0.05)$. To further prove our findings, miR-214 expression was also examined in several CRC cell lines. The CCD18Co cell, a cell line derived from colon tissue biopsy but not cancer tissue origin was used as the normal control. Based on our data, all CRC cell lines including DIFI, HCT-116, HT-29, SW620, Caco-2, CW-2 and RKO demonstrated a reduced expression of miR-214 compared with CCD-18Co cells $(\mathrm{P}<0.05)$ (Fig. 1B). Taken together, these data suggested a linkage of miR-214 downregulation and CRC.

\section{Identification of TFAM as a putative} target of $\mathrm{miR}-214$

To verify if TFAM could be a putative target, the 3'UTR of TFAM mRNA contained the putative binding sequence of miR-214 was constructed. Moreover, a mutated 3'UTR with two nucleotides replacement was constructed as well (Fig. 2A). Since HCT-116 and SW620 cells demonstrated the lowest expression level of miR-214, both UTRs were cloned to reporter vector and transfected to these two cell lines along with miR-214 mimic or miR-214 inhibitor. The data suggested that, in the mutated 3'UTR transfected cells, no significant changes of luciferase activity was observed when reporter was co-transfected with miR-214 mimic or miR-214 inhibitor. However, there

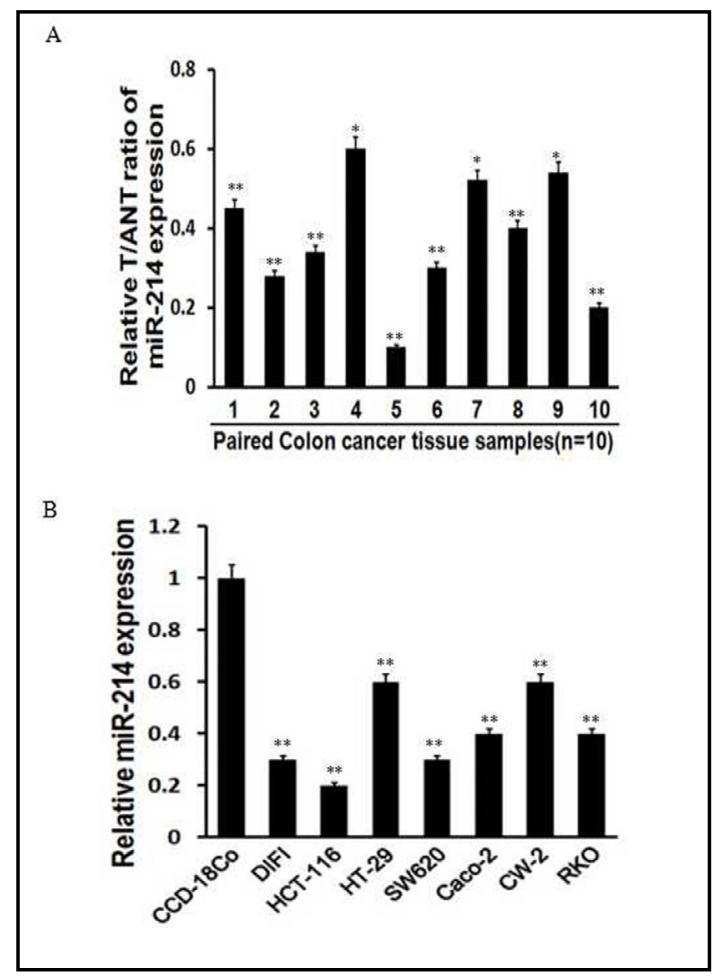

Fig. 1. The miR-214 expression level was reduced in CRC patients and CRC cell lines. A: miR-214 expression was evaluated in tumor samples from CRC patients. The value bellows 1 was defined as reduced expression. B: Evaluation of miR-214 expression in different CRC cell lines. All experiment was repeated for at least three times. ${ }^{*} \mathrm{P}<0.05$ and ${ }^{* *} \mathrm{P}<0.01$ indicated significant difference.

\section{KARGER}


Fig. 2. Identification of TFAM as a putative target of miR-214. A: Alignment of putative binding sites of miR-214 with TFAM 3'UTR as well as mutated TFAM 3' UTR. B: Luciferase reporter assay for miR214 inhibition of TFAM 3'UTR reporter and mutated TFAM 3' UTR mRNA. The TFAM 3'UTR and mutated 3'UTR reporter plasmids were co-transfected with miR-214 mimic or scramble control miRNA or miR-214 inhibitor in HCT-116 and SW620 cells for $48 \mathrm{~h}$, then cells were monitored for luciferase activity. For the qualification of luciferase activity, three repeated experiments have been conducted. *indicated significant difference $(\mathrm{P}<0.05)$.

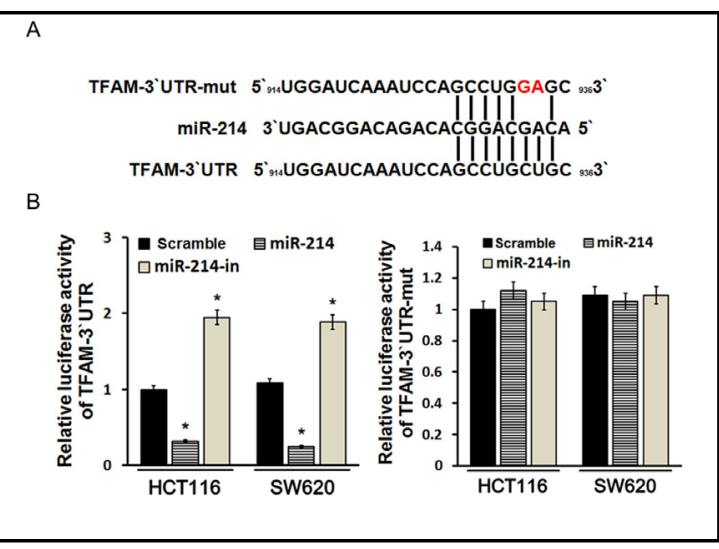

was a significant reduction in luciferase activity in both HCT-115 cells and SW620 cells when cells were co-transfected with miR-214 and TFAM 3'UTR reporter. Furthermore, transfection of miR-214 inhibitor in both cell lines resulted in the increased luciferase activity as expected.

On the other hand, to further confirm these findings using the reporter assay, endogenous TFAM expression was examined at both RNA and protein levels. It was found that, compared with HCT116 and SW620 cells transfected with scramble control, miR-214 inhibitor, TFAM mRNA expression was significantly upregulated by miR-214 mimic and reduced by miR-214 inhibitor respectively (Fig. $3 A$ ). Additionally, the results of TFAM protein level examined by western blot were consistent with the mRNA level detected by qPCR in each group. Taken together, above data suggested that TFAM was a putative target of miR-214 in CRC cells.

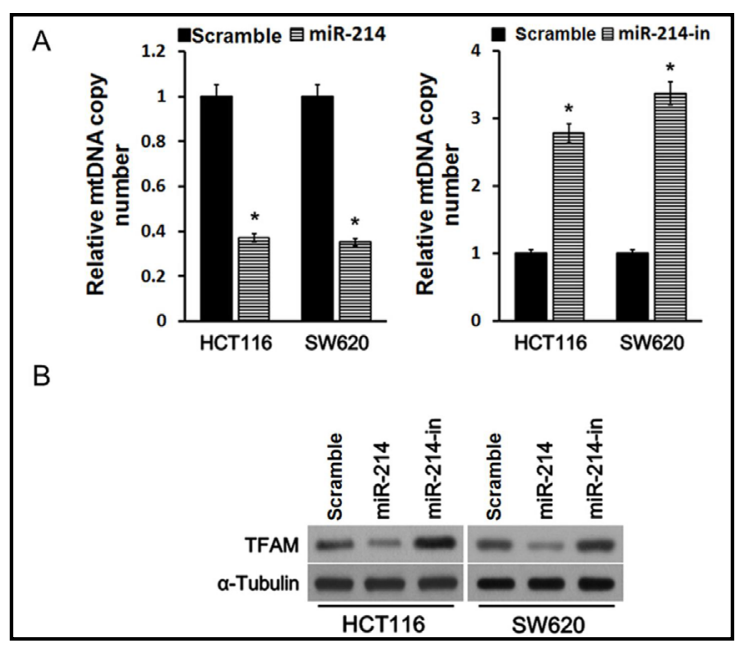

Fig. 3. The miR-214 mediated TFAM expression downregulation. A: CRC cells were transfected with miR-214 mimic, miR-214 inhibitor or scramble control and TFAM expression at the mRNA level was determined by qPCR. B. Western blot was used to test TFAM expression at the protein level in CRC cells transfected with miR214 mimic, miR-214 inhibitor or scramble control. Three individual experiments were repeated for each group. *indicated significant difference $(\mathrm{P}<0.05)$.

\section{Overexpression of miR-214 inhibited CRC cell proliferation}

The proliferation of CRC cells treated with miR-214 mimic or scramble control was monitored by MTT assay. Compared with the HCT-116 and SW620 transfected with scramble control, the miR-214 mimic transfection significantly inhibited the proliferation CRC cell lines HCT-116 and SW620 (Fig. 4A). To further confirm above findings, clonogenic cell survival assay was conducted as an alternative way to evaluate the capability of cell proliferation. A very strong inhibition of colonies formed by HCT-116 and SW620 was observed following miR-214 transfection (Fig. 4B and C). Additionally, as demonstrated in Fig. 4D, miR-214 overexpression decreased the percentage of HTC-116 cells $(71.36 \%)$ and SW620 cells (69.92\%) in the G1/G0 phase in comparison with scramble control transfected HTC-116 cells $(60.32 \%)$ and SW620 cells $(62.46 \%)$. The reduced cell percentage in G1/G0 phase could be the reason why cell proliferation was reduced in miR-214 transfected CRC cells. 
Overexpression of miR-214 resulted in the reduced $N F-\kappa B$ nuclear translocation and the inhibited $N F-\kappa B$ dependent gene expression

Also, the activation of NF- $\kappa B$ was monitored by reporter assay. After the transfection of both NF- $\kappa B$ reporter plasmids and control plasmids, miR214 mimic or miR-214 inhibitor was transfected to CRC cells as well. As shown in Fig. 5A, inhibition of TFAM via miR-214 significantly reduced luciferase activity in both HCT-116 cells and SW-620 cells. On the other hand, transfection of miR-214 inhibitor could result in the enhanced NF- $\kappa \mathrm{B}$ activity (Fig. 5A). In addition, to further verify the observations, nuclear translocation of NF- $\kappa \mathrm{B}$ was also examined. It was suggested that compared with the scramble control, miR-214 overexpression reduced nuclear translocation of NF- $\mathrm{BB}$ p65 subunit as expected while miR-214 inhibitor exerted the reversed effects (Fig. 5B). The expression level of the pro-apoptotic genes was examined after transfecting with miR-214 mimic and miR-214 inhibitor. As demonstrated in Fig. 5C, expression of NF- $\kappa B$ dependent genes such as myelocytomatosis oncogene (MYC), CyclinD1, B-cell lymphoma-extra large (Bcl-xL) and $\mathrm{X}$-linked inhibitor of apoptosis (XIAP) were significantly reduced in miR-214 overexpressed HCT-116 cells and SW620 cells. Taken together, these data suggested that overexpression of miR-214 could prevent nuclear translocation of NF- $\mathrm{B}$ as well as expression of NF- $\kappa \mathrm{B}$ dependent genes, which might be a reason for cell proliferation inhibition by miR-214.

\section{Down-regulation of TFAM induced by miR-214 was correlated with the reduced NF- $\kappa B$ nuclear translocation in $C R C$ patients \\ To confirm the correlation between}

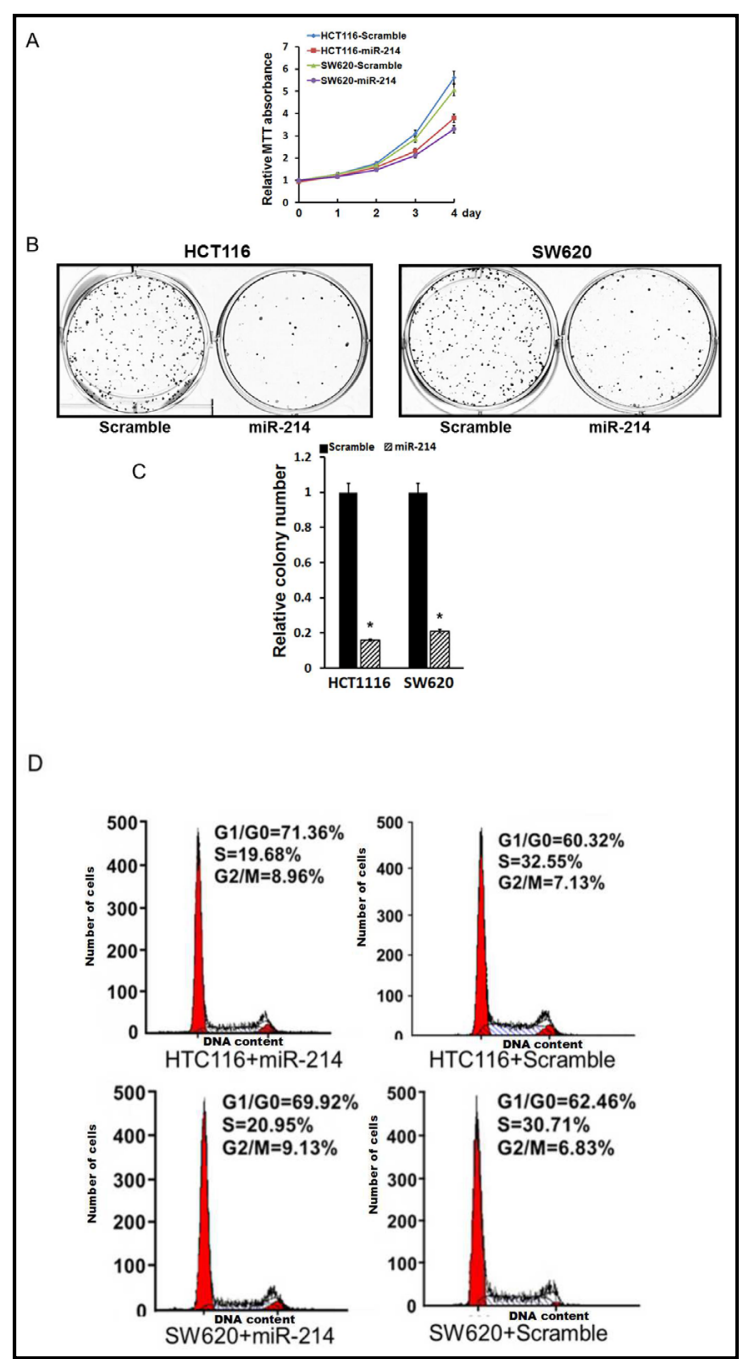

Fig. 4. Overexpression of miR-214 inhibited CRC cell proliferation. A: Cell proliferation was monitored by MTT assays in HTC-116 and SW620 cells transfected with miR-214 mimic, miR-214 inhibitor and scramble control. B: Clonogenic cell survival assay for HTC-116 and SW620 cells transfected with miR-214 mimic, miR214 inhibitor and scramble control. C: Quantification of clonogenic cell survival assay for HTC-116 and SW620 cells transfected with miR-214 mimic, miR-214 inhibitor and scramble control. Error bars represent standard deviation (SD) of three repeated experiments. *indicated significant difference $(\mathrm{P}<0.05)$. D: Flow cytometry analysis for cell cycles after transfection of miR-214 mimic, miR-214 inhibitor and scramble control in HTC-116 and SW620 cells.

TFAM down-regulation induced by miR-214 and the reduced NF- $\mathrm{BB}$ nuclear translocation in CRC cell lines, an investigation in tissue samples from CRC patients was also conducted. The data showed that all tissue samples obtained from CRC patients demonstrated higher TFAM expression and NF- $\mathrm{KB}$ nuclear translocation compared with normal tissue samples 
Fig. 5. Transfection of miR-214 mimic resulted in the reduced $N F-\kappa B$ nuclear translocation and inhibited NF- $\kappa$ B dependent gene expression. A. Luciferase assay for NF- $\kappa B$ activity in HTC-116 and SW620 cells transfected with miR-214 mimic, miR-214 inhibitor and scramble control. B: Nuclear translocation of NF- $\kappa \mathrm{B}$ p65 in HTC-116 and SW620 cells transfected with miR-214 mimic, miR-214 inhibitor and scramble control. C: The qPCR analysis for NF- $\mathrm{BB}$ dependent genes expression in HCT-116 and SW620 cells transfected with miR-214 mimic, miR-214 inhibitor and scramble control. Experiment for each sample was repeated for at least three times. *indicated significant difference $(\mathrm{P}<0.05)$.

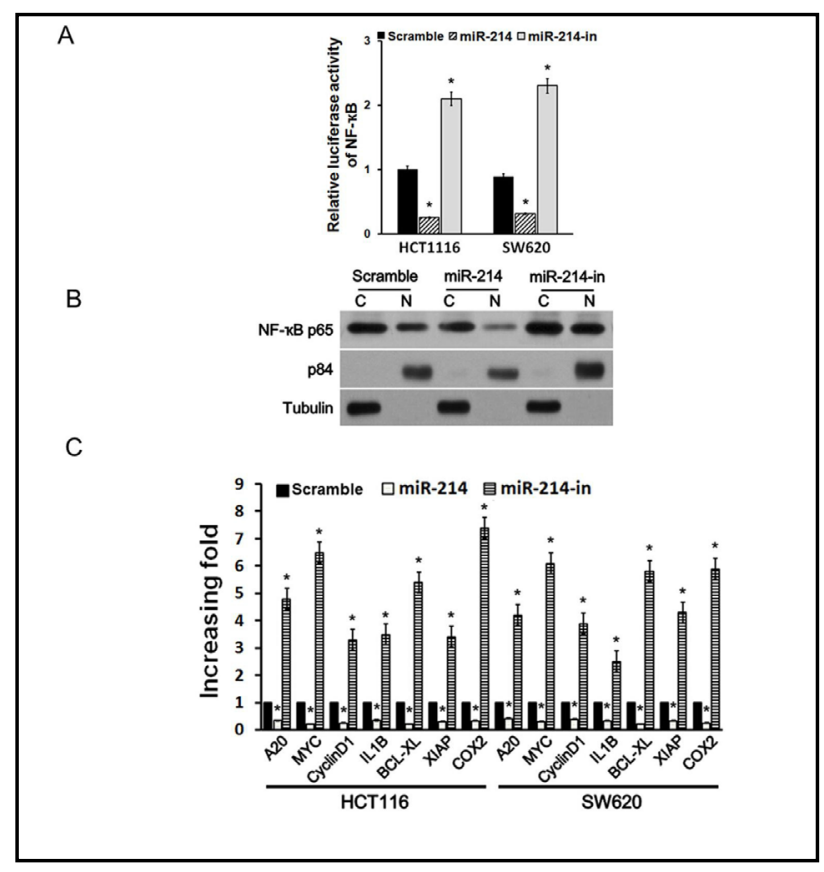

Fig. 6. miR-214 Down-regulation was correlated with the evaluated expression of TFAM and reduced $\mathrm{NF}-\kappa \mathrm{B}$ nuclear translocation in CRC patients. A. Nuclear translocation of NF- $\kappa$ B p65 in tumor tissue samples from CRC patients and healthy control. B: Relative expression of miR-214 in tumor tissue samples from CRC patients and healthy control. C: Correlation analysis between miR-214 expression and NF- $\kappa B$ expression and TFAM expression.

(Fig. 6A). miR-214 expression in CRC samples was downregulated with the increase of TFAM expression and NF- $\kappa \mathrm{B}$ translocation (Fig. 6B). A correlation analysis was also conducted and demonstrated in Fig. 6C which suggested a high correlation between miR-214 expression and TFAM expression and NF- $\mathrm{\kappa B}$ nuclear translocation. Taken together, it was

A

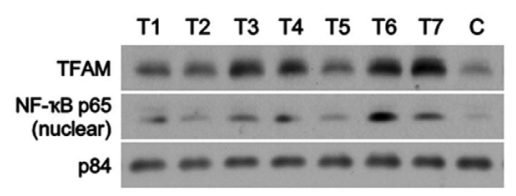

B

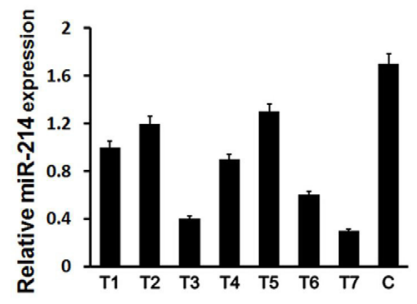

C
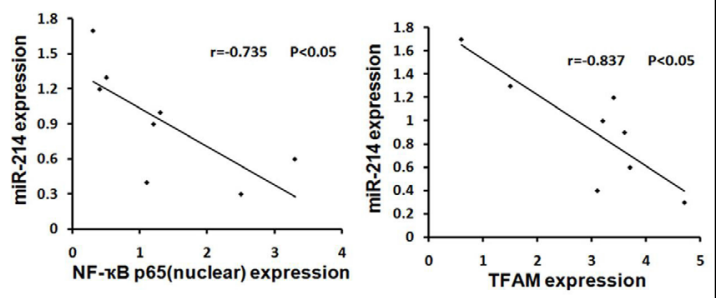
demonstrated that down-regulation of miR-214 in CRC was correlated with the evaluated TFAM level, indicating that miR-214 might be a potential marker for CRC prognosis or diagnosis.

\section{Discussion}

miR-214 was reported to act as a melano-miRNA which was involved in promoting the melanoma metastasis [26]. miR-214 expression was significantly increased in oral cancer 
and pancreatic cancer and its down-regulation resulted in the inhibition of cell apoptosis $[27,28]$. However, recent report suggested that miR-214 was down-regulated in cervical tumor tissues and cancer cells, and up-regulation of miR-214 leaded to the inhibition of cell growth, migration and invasion of cervical cancer cells and prompted the sensitivity to cisplatin $[29,30]$. For CRC, the role of miR-214 is still unknown. There, we conducted a screen of the miR-214 level in CRC tissue samples as well as CRC cells. Our study suggested that miR-214 expression was reduced in both tissue samples from CRC patients and CRC derived cell lines. Furthermore, this study discovered that miR-214 overexpression suppressed the proliferation of CRC cells.

Numerous studies have provided the evidence that miR-214 could changed biological functions of different cancer cells by its targets such as fibroblast growth factor receptor 1 (FGFR1), forkhead box M1 (FOXM1), Ras association domain family member 5 (RASSF5), Plexin-B1, phosphatase and tensin homolog (PTEN), and Ring finger and WD domain 2 (RFWD2) [27-35]. TFAM is a molecule which is essential for mtDNA replication and transcription, as well as mtDNA maintenance $[36,37]$. The TFAM-to-mtDNA ratio is critical for both mtDNA biogenesis and homeostasis, and even small variations in the TFAMto-mtDNA ratio may affect mtDNA replication [38]. In our study, we also examined the correlation between TFAM level and mtDNA and our data suggested that miR-214 level was also negatively correlated with mtDNA (data not shown), and miR-214 overexpression enhanced TFAM expression, which indicated a mechanism of TFAM involving in CRC.

Previous report demonstrated that down-regulation of TFAM in non-small cell lung cancer could activate reactive oxygen species (ROS)-mediated c-Jun N-terminal kinase(JNK)/ p38 mitogen-activated protein kinase (MAPK) signaling and reduce cellular bioenergetics which resulted in cell cycle arrest [16]. It was found in this study that TFAM expression could lead to the enhanced NF- $\mathrm{KB}$ activity as well as the promoted NF- $\mathrm{KB}$ dependent gene expression. In addition, the TFAM mRNA and protein levels were reported to be significantly elevated in a poorly differentiated rat hepatoma (Morris hepatoma) [39]. Increased TFAM expression levels were also observed in non-small cell lung cancer (NSCLC) and positively correlated to the malignancy and tumor grade as well [16].

The situation of CRC patients with higher expression of TFAM was worse than patients with low TFAM expression in tumor tissues [40], but the role TFAM played in CRC remains unclear. It was reported that TFAM depletion could result in upregulation of the levels of proapoptotic Bax, p21, tumor suppressor p53, and p-p53 (ser15), as well as poly ADP ribose polymerase (PARP), caspase 3 and caspase 9 cleavage [16]. In our study, TFAM knockdown by miR-214 mimic could reduce NF- $\mathrm{BB}$ nuclear translocation in vivo and in vitro. The assay conducted on cell lines also suggested that the reduced NF- $\kappa B$ nuclear translocation contributed to down-regulation of NF- $\mathrm{BB}$ depended genes. These data suggested a novel mechanism played by TFAM in CRC.

In conclusion, our study provided the clue that miR-214 expression was downregulated in CRC tissues and cell lines. Furthermore, miR-214 overexpression contributed to the enhanced TFAM expression and inhibited cell apoptosis. It was indicated that miR-214 and its target would be benefited for CRC treatment.

\section{Acknowledgements}

This study was supported by grants (81272637 and 81602598) awarded to Dr. Yulong He from the National Natural and Science foundation(NSFC) of China. The funder had no role in study design. KW, JM, YZ, KL and ZY were supported by NSFC grants No. 81272637 and supervised by the Dr. Yulong He. JC and KX were supported by NSFC grants No. 81602598 and supervised by Hongli Huang and Yulong He. 


\section{Cellular Physiology Cell Physiol Biochem 2018;49:545-554

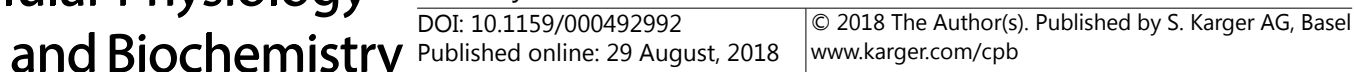 \\ Wu et al.: MicroRNA-214 Targeting TFAM}

\section{Disclosure Statement}

The authors declare to have no competing interests.

\section{References}

1 Anitha A, Maya S, Sivaram AJ, Mony U, Jayakumar R: Combinatorial nanomedicines for colon cancer therapy. Wiley Interdiscip Rev Nanomed Nanobiotechnol 2016;8:151-159.

2 PDQ Adult Treatment Editorial Board. Colon Cancer Treatment (PDQ(R)): PDQ Cancer Information Summaries [Internet]. Bethesda (MD): National Cancer Institute (US). 2002-2017.

$>3$ Lea MA: Recently identified and potential targets for colon cancer treatment. Future Oncol 2010;6:9931002.

$>4$ Watson AJ, Collins PD: Colon cancer: a civilization disorder. Dig Dis 2011;29(2):222-228.

-5 Ota M, Takamura N, Irimura T: Involvement of cell surface glycans in adhesion of human colon carcinoma cells to liver tissue in a frozen section assay: role of endo-beta-galactosidase-sensitive structures. Cancer Res 2000;60:5261-5268.

6 Ambros V: The functions of animal microRNAs. Nature 2004;431:350-355.

7 Jansson MD, Lund AH: MicroRNA and cancer. Mol Oncol 2012;6:590-610.

8 Akao Y, Nakagawa Y, Naoe T: let-7 microRNA functions as a potential growth suppressor in human colon cancer cells. Biol Pharm Bull 2006;29:903-906.

-9 Chen X, Guo X, Zhang H, Xiang Y, Chen J, Yin Y, Cai X, Wang K, Wang G, Ba Y, Zhu L, Wang J, Yang R, Zhang Y, Ren Z, Zen K, Zhang J, Zhang CY: Role of miR-143 targeting KRAS in colorectal tumorigenesis. Oncogene 2009;28:1385-1392.

10 Guo C, Sah JF, Beard L, Willson JK, Markowitz SD, Guda K: The noncoding RNA, miR-126, suppresses the growth of neoplastic cells by targeting phosphatidylinositol 3-kinase signaling and is frequently lost in colon cancers. Genes Chromosomes Cancer 2008;47:939-946.

11 Hofsli E, Sjursen W, Prestvik WS, Johansen J, Rye M, Trano G, Wasmuth HH, Hatlevoll I, Thommesen L: Identification of serum microRNA profiles in colon cancer. Br J Canc 2013;108:1712-1719.

12 Gao W, Wu MH, Wang N, Ying MZ, Zhang YY, Hua J, Chuan L, Wang YJ: Mitochondrial transcription factor A contributes to cisplatin resistance in patients with estrogen receptor-positive breast cancer. Mol Med Rep 2016;14:5304-5310.

13 Granados JB, Méndez JP, Feria-Bernal G, García-García E, Tejeda ME, Rojano-Mejía D, Tapia A, Canto P: Association of a TFAM haplotype with aggressive prostate cancer in overweight or obese Mexican Mestizo men. Urol Oncol 2017;35:111.e9-e14.

14 Peng H, Yang M, Chen ZY, Chen P, Guan CX, Xiang XD, Cai S, Chen Y, Fang X: Expression and methylation of mitochondrial transcription factor a in chronic obstructive pulmonary disease patients with lung cancer. PLoS One 2013;8:e82739.

15 Wen Z, Lei Z, Jin-An M, Xue-Zhen L, Xing-Nan Z, Xiu-Wen D: The inhibitory role of miR-214 in cervical cancer cells through directly targeting mitochondrial transcription factor A (TFAM). Eur J Gynaecol Oncol 2014;35:676-682.

16 Xie D, Wu X, Lan L, Shangguan F, Lin X, Chen F, Xu S, Zhang Y, Chen Z, Huang K, Wang R, Wang L, Song X, Liu Y, Lu B: Downregulation of TFAM inhibits the tumorigenesis of non-small cell lung cancer by activating ROS-mediated JNK/p38MAPK signaling and reducing cellular bioenergetics. Oncotarget 2016;7:1160911624.

17 Guo J, Zheng L, Liu W, Wang X, Wang Z, French AJ, Kang D, Chen L, Thibodeau SN: Frequent truncating mutation of TFAM induces mitochondrial DNA depletion and apoptotic resistance in microsatelliteunstable colorectal cancer. Cancer Res 2011;71:2978-2987.

18 Wen S, Gao J, Zhang L, Zhou H, Fang D, Feng S. p53 increase mitochondrial copy number via up-regulation of mitochondrial transcription factor A in colorectal cancer. Oncotarget 2016;7:75981-75995.

19 Mei H, Sun S, Bai Y, Chen Y, Chai R, Li H: Reduced mtDNA copy number increases the sensitivity of tumor cells to chemotherapeutic drugs. Cell Death Dis 2015;6:e1710.

20 Yang Z, Chen S, Luan X, Li Y, Liu M, Li X, Liu T, Tang H: MicroRNA-214 is aberrantly expressed in cervical cancers and inhibits the growth of HeLa cells. IUBMB life 2009;61:1075-1082. 


\section{Cellular Physiology Cell Physiol Biochem 2018;49:545-554 \begin{tabular}{l|l} 
DOI: 10.1159/000492992 & $\begin{array}{l}\text { O } 2018 \text { The Author(s). Published by S. Karger AG, Basel } \\
\text { www.karger.com/cpb }\end{array}$
\end{tabular}

Wu et al.: MicroRNA-214 Targeting TFAM

-21 Patel D, Nan Y, Shen M, Ritthipichai K, Zhu X, Zhang YJ: Porcine reproductive and respiratory syndrome virus inhibits type I interferon signaling by blocking STAT1/STAT2 nuclear translocation. J Virol 2010;84:11045-11055.

22 Patel D, Opriessnig T, Stein DA, Halbur PG, Meng XJ, Iversen PL, Zhang YJ: Peptide-conjugated morpholino oligomers inhibit porcine reproductive and respiratory syndrome virus replication. Antiviral Res 2008;77:95-107.

-23 Livak KJ, Schmittgen TD: Analysis of relative gene expression data using real-time quantitative PCR and the 2(-Delta Delta C(T)) Method. Methods (San Diego, CA) 2001;25:402-408.

-24 Nan Y, Wang R, Shen M, Faaberg KS, Samal SK, Zhang YJ: Induction of type I interferons by a novel porcine reproductive and respiratory syndrome virus isolate. Virology 2012;432:261-270.

-25 Munshi A, Hobbs M, Meyn RE: Clonogenic cell survival assay. Meth Mol Med 2005;110:21-28.

-26 Bar-Eli M: Searching for the 'melano-miRs': miR-214 drives melanoma metastasis. EMBO J 2011;30:18801881.

27 Li TK, Yin K, Chen Z, Bao Y, Zhang SX: MiR-214 regulates oral cancer KB cell apoptosis through targeting RASSF5. Genet Mol Res DOI: 10.4238/gmr16019327.

28 Zhang XJ, Ye H, Zeng CW, He B, Zhang H, Chen YQ: Dysregulation of miR-15a and miR-214 in human pancreatic cancer. J Hematol Oncol 2010;3:46.

29 Qiang R, Wang F, Shi LY, Liu M, Chen S, Wan HY, Li YX, Li X, Gao SY, Sun BC, Tang H: Plexin-B1 is a target of miR-214 in cervical cancer and promotes the growth and invasion of HeLa cells. Int J Biochem Cell Biol 2011;43:632-641.

-30 Wang JM, Ju BH, Pan CJ, Gu Y, Li MQ, Sun L, Xu YY, Yin LR: MiR-214 inhibits cell migration, invasion and promotes the drug sensitivity in human cervical cancer by targeting FOXM1. Am J Transl Res 2017;9:35413557.

-31 Schwarzenbach H, Milde-Langosch K, Steinbach B, Muller V, Pantel K: Diagnostic potential of PTENtargeting miR-214 in the blood of breast cancer patients. Breast Cancer Res Treat 2012;134:933-941.

-32 Yang H, Kong W, He L, Zhao JJ, O’Donnell JD, Wang J, Wenham RM, Coppola D, Kruk PA, Nicosia SV, Cheng JQ: MicroRNA expression profiling in human ovarian cancer: miR-214 induces cell survival and cisplatin resistance by targeting PTEN. Cancer Res 2008;68:425-433.

-33 Yang L, Ge D, Cao X, Ge Y, Chen H, Wang W, Zhang H: MiR-214 Attenuates Osteogenic Differentiation of Mesenchymal Stem Cells via Targeting FGFR1. Cell Physiol Biochem 2016;38:809-820.

-34 Yang TS, Yang XH, Wang XD, Wang YL, Zhou B, Song ZS: MiR-214 regulate gastric cancer cell proliferation, migration and invasion by targeting PTEN. Cancer Cell Int 2013;13:68.

-35 Zhang J, Su B, Gong C, Xi Q, Chao T: miR-214 promotes apoptosis and sensitizes breast cancer cells to doxorubicin by targeting the RFWD2-p53 cascade. Biochem Biophys Res Commun 2016;478:337-342.

-36 Little JP, Simtchouk S, Schindler SM, Villanueva EB, Gill NE, Walker DG, Wolthers KR, Klegeris A: Mitochondrial transcription factor A (Tfam) is a pro-inflammatory xtracellular signaling molecule recognized by brain microglia. Mol Cell Neurosc 2014;60:88-96.

-37 Ekstrand MI, Falkenberg M, Rantanen A, Park CB, Gaspari M, Hultenby K, Rustin P, Gustafsson CM, Larsson NG: Mitochondrial transcription factor A regulates mtDNA copy number in mammals. Hum Mol Genet 2004;13:935-944.

-38 Matsushima Y, Goto Y, Kaguni LS: Mitochondrial Lon protease regulates mitochondrial DNA copy number and transcription by selective degradation of mitochondrial transcription factor A (TFAM). Proc Natl Acad Sci U S A 2010;107:18410-18415.

-39 Dong X, Ghoshal K, Majumder S, Yadav SP, Jacob ST: Mitochondrial transcription factor A and its downstream targets are up-regulated in a rat hepatoma. J Biol Chem 2002;277:43309-43318.

40 Yoshida Y, Hasegawa J, Nezu R, Kim YK, Hirota M, Kawano K, Izumi H, Kohno K: Clinical usefulness of mitochondrial transcription factor A expression as a predictive marker in colorectal cancer patients treated with FOLFOX. Cancer Sci 2011;102:578-582. 